\title{
Direct numerical simulation of compressible turbulence in a counter-flow channel configuration
}

Arash Hamzehloo, ${ }^{1, *}$ David J. Lusher, ${ }^{2}{ }^{\dagger}$ Sylvain Laizet, ${ }^{1}$ and Neil D. Sandham ${ }^{2}$

${ }^{1}$ Turbulence Simulation Group, Department of Aeronautics, Imperial College London, London SWY 2AZ, United Kingdom

${ }^{2}$ Aerodynamics and Flight Mechanics Group, University of Southampton, Boldrewood Innovation Campus, Burgess Road, Southampton, SO16 7QF, United Kingdom 


\begin{abstract}
Counter-flow configurations, whereby two streams of fluid are brought together from opposite directions, are highly efficient mixers due to the high turbulence intensities that can be maintained. In this paper, a simplified version of the problem is introduced that is amenable to direct numerical simulation. The resulting turbulent flow problem is confined between two walls, with one non-zero mean velocity component varying in the space direction normal to the wall, corresponding to a simple shear flow. Compared to conventional channel flows, the mean flow is inflectional and the maximum turbulence intensity relative to the maximum mean velocity is nearly an order of magnitude higher. The numerical requirements and turbulence properties of this configuration are first determined. The Reynolds shear stress is required to vary linearly by the imposed forcing, with a peak at the channel centreline. A similar behaviour is observed for the streamwise Reynolds stress, the budget of which shows an approximately uniform distribution of dissipation, with large contributions from production, pressure-strain and turbulent diffusion. A viscous sublayer is obtained near the walls and with increasing Reynolds number small-scale streaks in the streamwise momentum are observed, superimposed on the large-scale structures that buffet this region. When the peak local mean Mach number reaches 0.55, turbulent Mach numbers of 0.6 are obtained, indicating that this flow configuration can be useful to study compressibility effects on turbulence.
\end{abstract}

\title{
I. INTRODUCTION
}

Counter-flow shear layers, also known as counter-current flows, have been previously recognised as efficient flow configurations for mixing [1-4], thrust vectoring (flow control) [5-7] and combustion [8, 9]. Strykowski and Wilcoxon [2] showed that counter-current configurations could be used to control axisymmetric jet flows and enhance their mixing characteristics using global oscillations produced by self-excitation within shear layers. Strykowski and colleagues $[8,9]$ also showed that a counter-current flow configuration can be used in swirl combustors to efficiently control the turbulent burning velocity (flame speed) and reduce pollutant emissions. The use of counter-flow configurations for fundamental flow studies was first recognised by Humphrey and $\mathrm{Li}$ [1] and later by various researchers including most notably Forliti et al. [3] who studied planar shear layers. A shear layer develops

* Arash Hamzehloo: a.hamzehloo@imperial.ac.uk

$\dagger$ Current address: Shocks, Solitons, and Turbulence Unit. Okinawa Institute of Science and Technology. 1919-1 Tancha Onna, Okinawa, Japan. 
when two fluid streams interact at a different velocity. Shear flows are typically quantified by the velocity ratio $(r)$ and the normalised velocity ratio $(\lambda)$ defined as $r=U_{2} / U_{1}$ and $\lambda=\left(U_{1}-U_{2}\right) /\left(U_{1}+U_{2}\right)[3]$. Here, $U_{1}$ and $U_{2}$ denote the velocity magnitude of the two flow streams (primary and secondary flows, respectively). A counter-flow forms when $r<0$. It has been shown theoretically [10] and experimentally [11] that a counter-flow shear layer experiences transition from convective to absolute (global) instability when the secondary reverse stream achieves a velocity magnitude of around $13 \%$ or higher of that of the primary stream i.e. $|r|>0.136$ or $\lambda>1.315[3]$.

Humphrey and $\mathrm{Li}$ [1] studied vortex generation and collapse mechanisms at the interface of two counter-flowing streams initially separated by splitter plates in a channel configuration $(r=-1.0)$ in the laboratory. The two flow streams were laminar with the Reynolds number, based on the channel width and the mass average velocity in one of the flows, ranging from 370 to 1000 . The use of such counter-flow channel configuration was found to be a relatively simple but effective way to study vortex-induced mixing. However, their set-up resulted in a stagnation zone which eventually disrupted the shear layer. Later, Humphrey et al. [4] conducted a numerical study on counter-flow shear layers based on the experimental setup of Humphrey and Li [1]. They investigated the effect of the Reynolds number using symmetric and antisymmetric counter-flowing velocity profiles. It was concluded that the convective transport at such Reynolds numbers was not able to remove vortical structures due to their fast generation and amplification rate which consequently resulted in rotational flow instabilities and a periodic breakdown.

Momentum-driven counter-flows, like the ones studied by Humphrey and Li [1], have proven to be experimentally difficult to create. Therefore, suction-driven approaches, which typically utilise suction-type centrifugal fans to drive the secondary backward stream, have been used to create counter-flow shear layers as, for example, discussed in $[3,11]$. Forliti et al. [3] were the first to study the characteristics of planar counter-flows. In their wind tunnel setup, a splitter plate separated the primary stream and the suction-driven secondary stream. Velocity ratios up to $r=-0.3$ with $U_{1}=31 \mathrm{~m} / \mathrm{s}$ were investigated. A critical normalised velocity ratio of $\lambda=1.3$ was found for the onset of the transitional instability which was in line with earlier theoretical predictions and experimental measurements. It was concluded that locally unstable flows trigger the global instability in counter-flow shear layers [3].

A further potential application of counter-flow shear layers is in the study of compressibility, which has major effects on the turbulent characteristics of fluid flows and is typically 
manifested through, among others, reduction in turbulence production, acoustic/entropy oscillations, shear layers growth suppression, shock formation and non-linear shock-turbulence interactions [12-15]. Many aspects of the complex interactions between compressibility effects and turbulence structures are still not thoroughly understood. Traditional free shear layers (such as jets, wakes and mixing layers) and Poiseuille/Couette type flows (such as channel flows) have been utilized to study compressible turbulence [13, 15, 16]. Spatiallydeveloping mixing layer simulations are computationally expensive and are sensitive to farfield and inflow/outflow boundary conditions [16, 17], but the basic compressibility effects are captured in temporal simulation as, for example, discussed by Vreman et al. [18]. A drawback of the temporal approach is that the shear layer thickens as time progresses and large structures rapidly fill the computational domain. Using much larger domains is then inefficient for the early development stages of the flow. On the other hand, Poiseuille/Couette type flows are relatively efficient to compute [19-21] and can achieve high Reynolds numbers for much lower cost than what is typically required for a spatially-developing boundary layer [20]. Conventional channel flow is limited in terms of the fluctuating Mach number that can be reached, due to the fact that the mean flow becomes hot, with the associated increase in the speed of sound. A counter-flow channel configuration can potentially overcome the barriers of the above-mentioned flows. Specifically, it retains a statistically stationary one-dimensional solution, in common with conventional channel flows, but contains an inflectional mean flow, representative of free shear layers.

Another version of channel flow that produces counter-flows of the kind considered here is from Waleffe [22]. That flow was devised to study exact solutions of the Navier-Stokes equations in a confined setting and in that context it was important that the base flow was linearly stable. In the present study, however, we introduce a new wall-bounded counter-flow channel configuration and argue that, in contrast, its unstable base flow is more relevant to free shear layers (jets, wakes and mixing layers) found in practice. Here, there are different questions about organised structures that make such flows interesting from a theoretical perspective. A recent example is the work of Mantič-Lugo and Gallaire [23, 24] that proposed a self-consistent model of structures in backward-facing step configuration in which the timeaveraged flow is unstable.

In the present numerical study we first introduce the new counter-flow configuration and the Direct Numerical Simulation (DNS) approach used to study it. Since this is the first time such flows have been simulated, we spend some time on grid and domain size requirements 
and explain the flow structure (section III). Then in section IV we increase the Reynolds number and examine the flow near the walls, where the near-wall turbulence is buffeted by large shear layer structures. Finally, in section $\mathrm{V}$ the Mach number is increased to check whether the configuration can sustain high turbulent Mach numbers.

\section{METHODOLOGY}

\section{A. Configuration}

DNS of compressible turbulent counter-flow channels with different domain sizes of the form $L_{x} H \times 2 H \times L_{z} H$ are conducted. $H$ denotes the channel half height and is set to $H=1$ in the current study. $L_{x}$ and $L_{z}$ are the domain lengths in the $x$ (streamwise) and $z$ (spanwise) directions, respectively. Dimensionless streamwise and spanwise dimensions range from 6 to 24 and 3 to 12, respectively. Figure 1 shows a schematic of the counter-flow channel configuration introduced in this study. The streamwise and spanwise boundaries are periodic, while isothermal $\left(T_{w}=1.0\right)$ no-slip walls are assigned to the boundaries in the normal direction $(y)$. In order to accurately resolve the near wall region, the grid is stretched in the $y$ direction using a stretching function with a stretching factor of 1.7 as discussed in [25]. A tangent hyperbolic forcing function is used to drive the flow in opposite directions on the upper and lower halves of the channel. This consequently forms an antisymmetric mean "shear" velocity profile as shown schematically by the blue profile in Figure 1.

Unless otherwise stated, simulations are initialized using an approach originally developed for DNS of conventional channel flows as discussed in [25]. Specifically, fluctuations based

on sine and cosine disturbances are superimposed over a mean classical turbulent velocity profile in the $x$ direction and in other directions only the sine and cosine disturbances are imposed. All simulations are initially conducted for relatively long transient periods (as long as $t=400$ ) to obtain fully developed counter-flows before collecting the statistics for an additional period of $t=1000$.

\section{B. Governing equations}

The dimensionless governing equations of a compressible Newtonian fluid flow that conserve mass, momentum and energy are given as: 


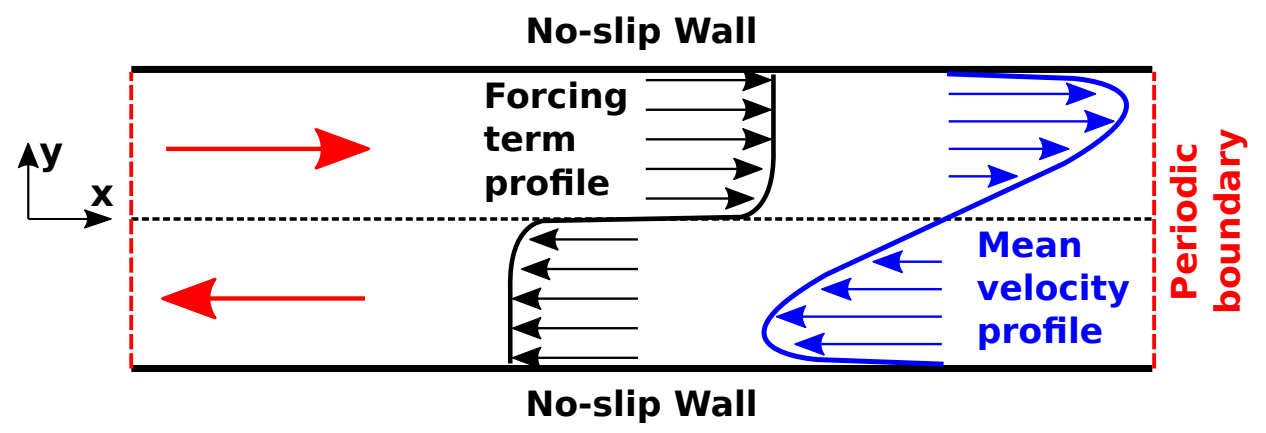

FIG. 1. 2D schematic of the 3D counter-flow channel configuration. The streamwise and spanwise (in-plane, in the $z$ direction) boundaries are periodic. The top and bottom boundaries are isothermal no-slip walls. The red arrows show the theoretical directions of the mean flow in the upper and lower halves of the channel. Profiles of the hyperbolic tangent forcing term $\left(c_{1}\right)$ and the mean streamwise velocity are shown by the black and blue arrows, respectively. The black dashed line shows the channel centreline at $y=0$. An alternative coordinate in the $y$ direction relative to the wall will be denoted $\tilde{y}$ (with $\tilde{y}=0$ is at the wall) and will be used to compute the normalised wall distance $\left(\tilde{y}^{+}\right)$.

$$
\begin{gathered}
\frac{\partial \rho}{\partial t}+\frac{\partial}{\partial x_{j}}\left(\rho u_{j}\right)=0 \\
\frac{\partial \rho u_{i}}{\partial t}+\frac{\partial}{\partial x_{j}}\left(\rho u_{i} u_{j}+p \delta_{i j}-\tau_{i j}\right)+c_{j} \delta_{i j}=0, \\
\frac{\partial \rho E}{\partial t}+\frac{\partial}{\partial x_{j}}\left(\rho E u_{j}+u_{j} p+q_{j}-u_{i} \tau_{i j}\right)+c_{j} u_{j}=0,
\end{gathered}
$$

where $\rho$ represents the density, $u_{i}(i=1,2,3)$ denotes the velocity component $(u, v$ and $w$, respectively) in the $i^{\text {th }}$ direction ( $x, y$ and $z$, respectively), $E$ is the total energy, and $p$ and $\delta$ denote the pressure and the Kronecker delta, respectively. The equations are solved in three spatial dimensions $x_{i}(i=1,2,3)$. The forcing term $c_{j}$ drives the flow, with a value of $c_{1}=-c_{0} \tanh \left(a x_{2}\right)$ in the $x_{1}$ direction and zero in other directions. The maximum value is set as $c_{0}=1$. Here $-1<x_{2}<1$, therefore equivalent driving forces are applied in opposite directions to the upper and lower halves of the domain which consequently result in the formation of a shear-forcing or counter-flow condition with a constant flow rate. The coefficient $a$ in the forcing term is positive with a value of $a=100$ in the present study. The viscous stress tensor $\left(\tau_{i j}\right)$ and the heat flux $\left(q_{j}\right)$ are defined as: 


$$
\begin{gathered}
\tau_{i j}=\frac{\mu}{R e}\left(\frac{\partial u_{i}}{\partial x_{j}}+\frac{\partial u_{j}}{\partial x_{i}}-\frac{2}{3} \frac{\partial u_{k}}{\partial x_{k}} \delta_{i j}\right), \\
q_{j}=\frac{-\mu}{(\gamma-1) M^{2} \operatorname{Pr} \operatorname{Re}} \frac{\partial T}{\partial x_{j}}
\end{gathered}
$$

where $\mu$ denotes the dynamic viscosity, $T$ is the temperature, $\gamma$ is the ratio of specific heats with a value of $\gamma=1.4$ here and $\operatorname{Pr}$ is the Prandtl number with a value of $\operatorname{Pr}=0.7$ in this study. Also, Re and $M$ denote the Reynolds and Mach numbers based on a reference

velocity deduced from the forcing as $u_{\mathrm{ref}}^{*}=\sqrt{c_{0} H /\langle\rho\rangle_{b}}$, where $\langle\rho\rangle_{b}$ is the bulk-averaged density, together with the channel half height $H$ and the wall temperature and viscosity. Angle brackets \langle\rangle denote averages over the homogeneous spatial directions $(x$ and $z)$ and time throughout this study. The additional subscript $b$ here denotes an additional average over $y$. All quantities are non-dimensionalised by the bulk-averaged density, the reference velocity $\left(u_{\mathrm{ref}}^{*}\right)$ and the wall temperature and viscosity. The dynamic viscosity is calculated as $\mu=T^{0.7}$. The temperature is calculated as $T=p \gamma M^{2} / \rho$. Here the pressure of an ideal Newtonian fluid is obtained using an equation of state as:

$$
p=(\gamma-1)\left(\rho E-\frac{1}{2} \rho u_{i} u_{i}\right)
$$

A fourth order finite-difference central scheme is used to discretise the governing equations which are recast in split skew-symmetric formulations to improve numerical stability [26, 27]. For non-periodic boundaries a fourth order one-sided boundary closure is used [28, 29]. A low-storage three-stage explicit Runge-Kutta scheme [30] is used to advance the solution in time.

\section{Computational framework}

The governing equations are discretised and solved using OpenSBLI, a Python-based automatic source code generation and parallel computing framework for finite difference discretisation [31, 32]. It automatically generates $\mathrm{C}$ codes for the Oxford Parallel library for Structured mesh solver (OPS), an embedded Domain Specific Language (DSL) with associated libraries and pre-processors to generate parallel executables for applications on multi-block structured meshes [33, 34]. OPS can target various computational architectures based on CUDA or OpenCL for GPUs, MPI or OpenMP for CPUs and CUDA-MPI for mul- 
tiple GPUs [34]. A comprehensive discussion on the automatic derivation Python interface of OpenSBLI and the automatic procedure of the OPS C code generation can be found in $[31,32]$. The results presented in this numerical study are obtained using single (CUDA) and multiple (MPI+CUDA) NVIDIA P100 GPUs.

\section{Problem specifications}

In the present study various Mach and Reynolds number values, in addition to different channel sizes and grid resolutions, are investigated. A list of test cases studied here is presented in table I. First, the effect of the domain size and the grid resolution on the turbulence characteristics and flow structures are evaluated with $M=0.1$ and $R e=400$. The height of the channel is kept constant and different domain sizes have different lengths in the streamwise $x$ and spanwise $z$ directions as $6 \times 2 \times 3,12 \times 2 \times 6$ and $24 \times 2 \times 12$. For the domain size study, a similar grid resolution is maintained between the different cases (cases 1, 4 and 5 of table I) as shown in table II based on the normalised cell sizes in different directions. The normalised wall distance value $\left(\tilde{y}^{+}\right)$is defined as $\tilde{y}^{+}=\tilde{y} R e_{\tau}$, where $\tilde{y}$ is the coordinate in the $y$ direction relative to the wall. The normalised cell sizes in different directions, $\Delta x^{+}, \Delta \tilde{y}^{+}$(the height of the first grid point above the wall) and $\Delta z^{+}$, are evaluated in a similar way to $\tilde{y}^{+}$. The friction Reynolds number is defined as

$R e_{\tau}=\left\langle\rho_{\text {wall }}\right\rangle u_{\tau} H /\left\langle\mu_{\text {wall }}\right\rangle$ where $u_{\tau}=\sqrt{\left\langle\tau_{\text {wall }}\right\rangle /\left\langle\rho_{\text {wall }}\right\rangle}$. The effect of the grid resolution is studied for the $12 \times 2 \times 6$ domain size based on test cases 2,3 and 4 of table I.

The effect of the Reynolds number is investigated by conducting DNS of $M=0.1$ counterflow cases with $R e=800$ and $R e=1600$ (cases 9 and 10) on the $6 \times 2 \times 3$ domain size. Finally, the friction Mach number is increased from $M=0.1$ to 0.4 to introduce higher compressibility effects. Various time steps from $\Delta t=5 \times 10^{-5}$ to $2 \times 10^{-4}$ are used depending on the particular case as provided in table I. In tables I and II, subscript $p$ denotes the peak values of the flow quantities listed. Also, $\langle u\rangle_{b}$ is the bulk velocity (the average of the absolute mean streamwise velocity over the channel).

It should be noted that, in this numerical study, the single prime ' denotes the turbulent fluctuations which for an arbitrary flow quantity $(\phi)$ is defined as $\phi^{\prime}=\phi-\langle\phi\rangle$. Moreover, for the higher Mach number case, the Favre average is defined as $\{\phi\}=\langle\rho \phi\rangle /\langle\rho\rangle$ and the double prime " denotes the turbulent fluctuation with respect to the Favre average defined as $\phi^{\prime \prime}=\phi-\{\phi\}$. For the Reynolds stresses, the Favre average is related to the Reynolds 
average as $\langle\rho\rangle\left\{u_{i}^{\prime \prime} u_{j}^{\prime \prime}\right\}=\left\langle\rho u_{i} u_{j}\right\rangle-\langle\rho\rangle\left\langle u_{i}\right\rangle\left\langle u_{j}\right\rangle$. Also, the mean Mach number is defined as $\langle M\rangle=\sqrt{\langle u\rangle^{2}+\langle v\rangle^{2}+\langle w\rangle^{2}} /\langle a\rangle$, where $a$ is the local speed of sound, while the turbulent Mach number is defined as $M_{t}=\sqrt{\left\langle u^{\prime} u^{\prime}\right\rangle+\left\langle v^{\prime} v^{\prime}\right\rangle+\left\langle w^{\prime} w^{\prime}\right\rangle} /\langle a\rangle$.

TABLE I. DNS counter-flow channel cases.

\begin{tabular}{cccccccccc}
\hline Case & $M$ & $R e$ & Domain & Grid & $\Delta \mathrm{t}\left(\times 10^{-4}\right)$ & $\langle u\rangle_{b}$ & $\langle u\rangle_{p}$ & $\langle M\rangle_{p}$ & $M_{t_{p}}$ \\
\hline 1 & 0.1 & 400 & $6 \times 2 \times 3$ & $120 \times 151 \times 100$ & 2.0 & 1.261 & 1.885 & 0.182 & 0.211 \\
2 & 0.1 & 400 & $12 \times 2 \times 6$ & $192 \times 151 \times 160$ & 2.0 & 1.400 & 2.147 & 0.206 & 0.213 \\
3 & 0.1 & 400 & $12 \times 2 \times 6$ & $240 \times 131 \times 200$ & 2.0 & 1.410 & 2.162 & 0.207 & 0.213 \\
4 & 0.1 & 400 & $12 \times 2 \times 6$ & $240 \times 151 \times 200$ & 2.0 & 1.407 & 2.159 & 0.207 & 0.213 \\
5 & 0.1 & 400 & $24 \times 2 \times 12$ & $480 \times 151 \times 400$ & 2.0 & 1.391 & 2.130 & 0.204 & 0.214 \\
6 & 0.4 & 400 & $12 \times 2 \times 6$ & $240 \times 151 \times 200$ & 0.5 & 1.364 & 2.047 & 0.556 & 0.595 \\
7 & 0.1 & 800 & $6 \times 2 \times 3$ & $240 \times 301 \times 200$ & 1.0 & 1.359 & 2.069 & 0.196 & 0.216 \\
8 & 0.1 & 1600 & $6 \times 2 \times 3$ & $480 \times 601 \times 400$ & 0.5 & 1.401 & 2.124 & 0.198 & 0.217 \\
\hline
\end{tabular}

TABLE II. Computational parameters of the counter-flow cases.

\begin{tabular}{ccccccccc}
\hline Case & $M$ & $R e$ & $R e_{\tau}$ & $u_{\tau}$ & $\Delta x^{+}$ & $\Delta \tilde{y}^{+}$ & $\Delta z^{+}$ & $\langle u\rangle_{p}^{+}$ \\
\hline 1 & 0.1 & 400 & 124.474 & 0.286 & 6.223 & 0.385 & 3.734 & 6.578 \\
2 & 0.1 & 400 & 130.841 & 0.299 & 8.177 & 0.404 & 4.906 & 7.182 \\
3 & 0.1 & 400 & 131.376 & 0.299 & 6.568 & 0.470 & 3.941 & 7.224 \\
4 & 0.1 & 400 & 131.482 & 0.299 & 6.574 & 0.406 & 3.944 & 7.209 \\
5 & 0.1 & 400 & 130.745 & 0.298 & 6.537 & 0.404 & 3.922 & 7.145 \\
6 & 0.4 & 400 & 193.835 & 0.210 & 9.691 & 0.599 & 5.815 & 9.716 \\
7 & 0.1 & 800 & 225.726 & 0.251 & 5.643 & 0.345 & 3.385 & 8.218 \\
8 & 0.1 & 1600 & 407.819 & 0.219 & 5.097 & 0.310 & 3.058 & 9.671 \\
\hline
\end{tabular}

\section{FLOW STRUCTURES AND STATISTICS}

\section{A. Flow structure}

Contours of the instantaneous streamwise velocity fluctuation for the counter-flow case with the $24 \times 2 \times 12$ domain size are plotted on three $x-z$ planes at different heights in 
figure 2. Also, contours of the instantaneous streamwise velocity $(u)$ for the same counterflow case on different $x-y$ and $y-z$ planes are shown in figure 3. The horizontal planes of figure 2 with $y=0, y \approx 0.791$ and $y \approx 0.905$ cut through (a) the channel centreline, (b) the peak of the mean streamwise velocity and $(\mathrm{c})$ the near-wall region $\left(\tilde{y}^{+} \approx 12\right)$, respectively. At $y=0$, elongated streaks with opposing flow directions are seen. Towards the upper wall the streaks disappear, in contrast to conventional channel flow where streamwise streaks would be observed near the wall. The high velocity of the fluctuating centreline streaks is associated with significant ejections of the flow from one half into the other half of the counter-flow channel, something which is clear in the velocity contours shown in figure 3 and also the Q-criterion iso-surfaces shown in figure 4. From the iso-surfaces of figure 4, which are coloured by the magnitude of the instantaneous streamwsie velocity, it is clear that the counter-flow configuration produces highly complex three-dimensional vortical turbulent structures particularly in the vicinity of the channel centreline. The existence of the elongated streamwise streaks seen in $y=0$ snapshot of figure 2 are also recognisable from the spatial distribution of the $\mathrm{Q}$ iso-surfaces of figure 4 . In fact, such complex vortical structures are mainly generated near the channel centreline, where the counter-flowing streaks break down. A level of spanwise flow coherence is seen in figure 2 (also seen in similar plots for the case with a $12 \times 2 \times 6$ domain size that is not shown here). Iso-surfaces of the pressure (not shown here) revealed that the $6 \times 2 \times 3$ case exhibited a quite different flow pattern with a large vortical structure spanning the entire domain but this was not clearly present for wider domains. Quantitative comparisons will be presented in the next section, but there is a substantial domain-dependency in the flow structures between $6 \times 2 \times 3$ and $12 \times 2 \times 6$ domain sizes.

Figure 5 shows the two-point correlations for the fluctuating part of the velocity components as a function of the spanwise distance $(z)$ at different channel heights for the test case with a $24 \times 2 \times 12$ domain size. The correlations plotted in figure 5 are accumulated and averaged over a period of $t \approx 120$. The values are also spatially averaged over all grid points in the $x$ direction at each $y$ location. The two-point correlation is evaluated in the spanwise direction with a reference point at $z=0$ as [35]:

$$
R(z)_{u_{i}^{\prime} u_{i}^{\prime}}=\frac{\left\langle u_{i}^{\prime}(0) u_{i}^{\prime}(z)\right\rangle}{\sqrt{\left\langle u_{i}^{\prime 2}(0)\right\rangle\left\langle u_{i}^{\prime 2}(z)\right\rangle}} .
$$

All two-point correlations decay to zero which indicate that the size of the channel is 


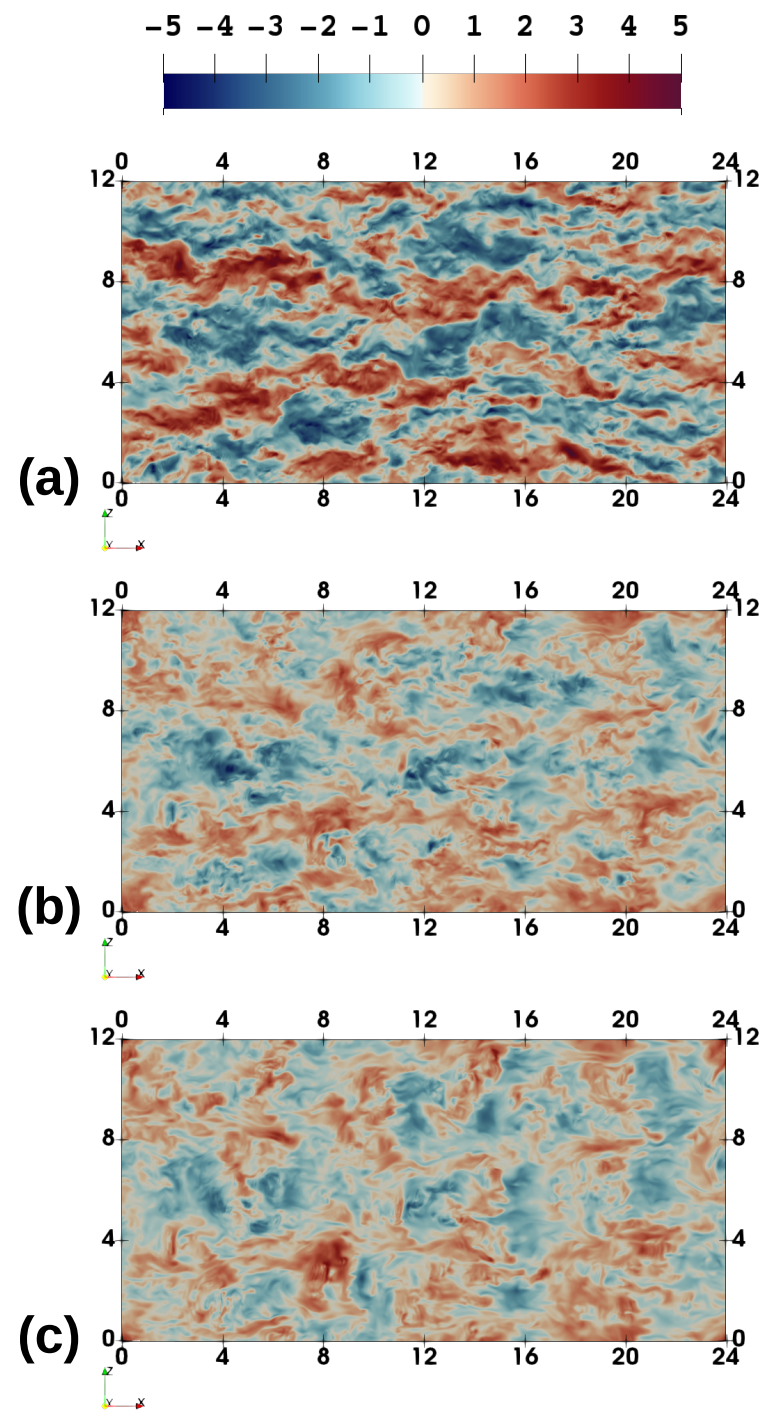

FIG. 2. Instantaneous field of the streamwise velocity fluctuation $\left(u^{\prime}=u-\langle u\rangle\right)$ on $x-z$ planes at different channel heights for the counter-flow cases with $M=0.1, R e=400$ and a $24 \times 2 \times 12$ domain size at $t=1400$. (a): $y=0.0,(\mathrm{~b}): y \approx 0.791\left(\tilde{y}^{+} \approx 27\right)$ and $(\mathrm{c}): y \approx 0.905\left(\tilde{y}^{+} \approx 12\right)$.

large enough in the spanwise direction [36]. The case with a $12 \times 2 \times 6$ domain size exhibits a very close flow characteristics to the case with a $24 \times 2 \times 12$ domain size in terms of the flow structures, mean and turbulent flow statistics as we shall see in the next subsection. Therefore, it is selected to investigate the effect of the Reynolds and Mach numbers with a reasonable computational cost in this study. 


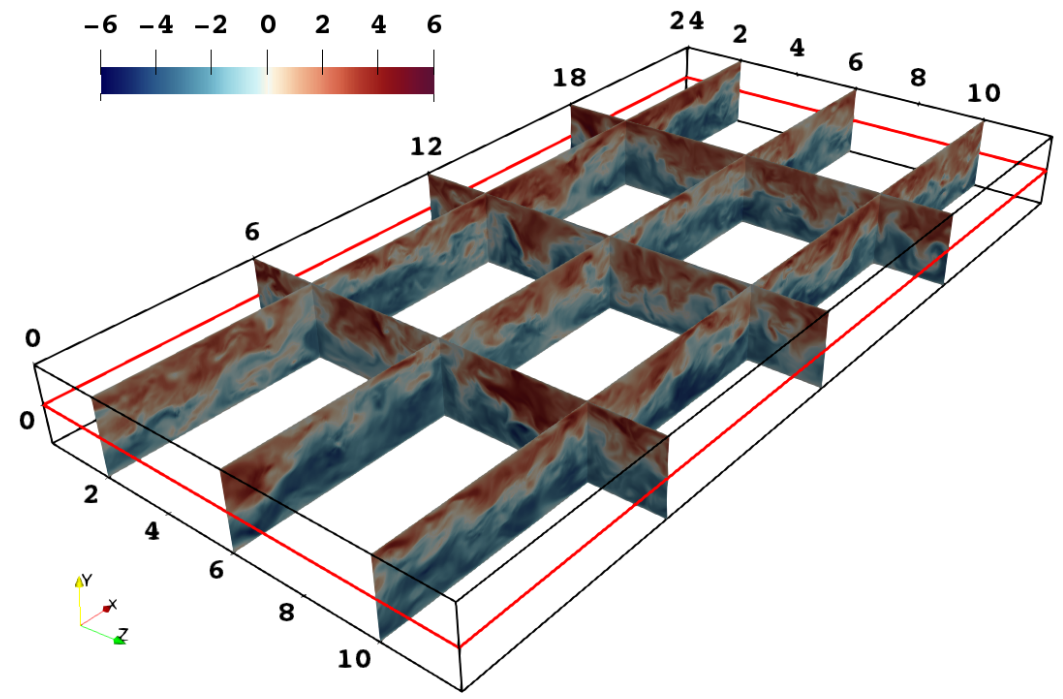

FIG. 3. Instantaneous field of the streamwise velocity $(u)$ on various planes for the counter-flow case with $M=0.1, R e=400$ and a $24 \times 2 \times 12$ domain size at $t=1400$. The red lines show the outlines of the $x-z$ plane cutting through the channel centreline (the figure shows the full height of the channel from $y=-1$ to $y=1)$.

\section{B. Mean flow and turbulence statistics}

In this subsection the effect of the domain size and grid resolution is investigated using the test cases with $M=0.1$ and $R e=400$ (cases 1 to 5 of table I). Figure 6 shows a direct comparison between those cases based on the mean flow streamwise velocity and the Reynolds stresses. Please note that in the graphs provided in this paper, the markers (if applicable) are shown for a limited number of grid points as specified in the captions. Except for the test case with the smallest domain size $(6 \times 2 \times 3)$ the other test cases exhibit very close profiles and peak values. Case 1 with a domain size of $6 \times 2 \times 3$ has lower peak streamwise velocity and mean Mach number by around $12.7 \%$ and $12.0 \%$, respectively compared to case 4 which has a larger domain size $(12 \times 2 \times 6)$ but a similar grid resolution. With respect to the turbulent Mach number, all cases exhibit very close profiles and peak values around 0.21 as provided in table I. With the peak mean Mach number also around 0.21, these cases can be considered effectively incompressible, albeit with approximately a $10 \%$ variation in mean density and temperature that would need to be taken into account in modelling this flow. Over the core of the channel (out to $y=0.75$ ), the mean velocity shows an approximately 


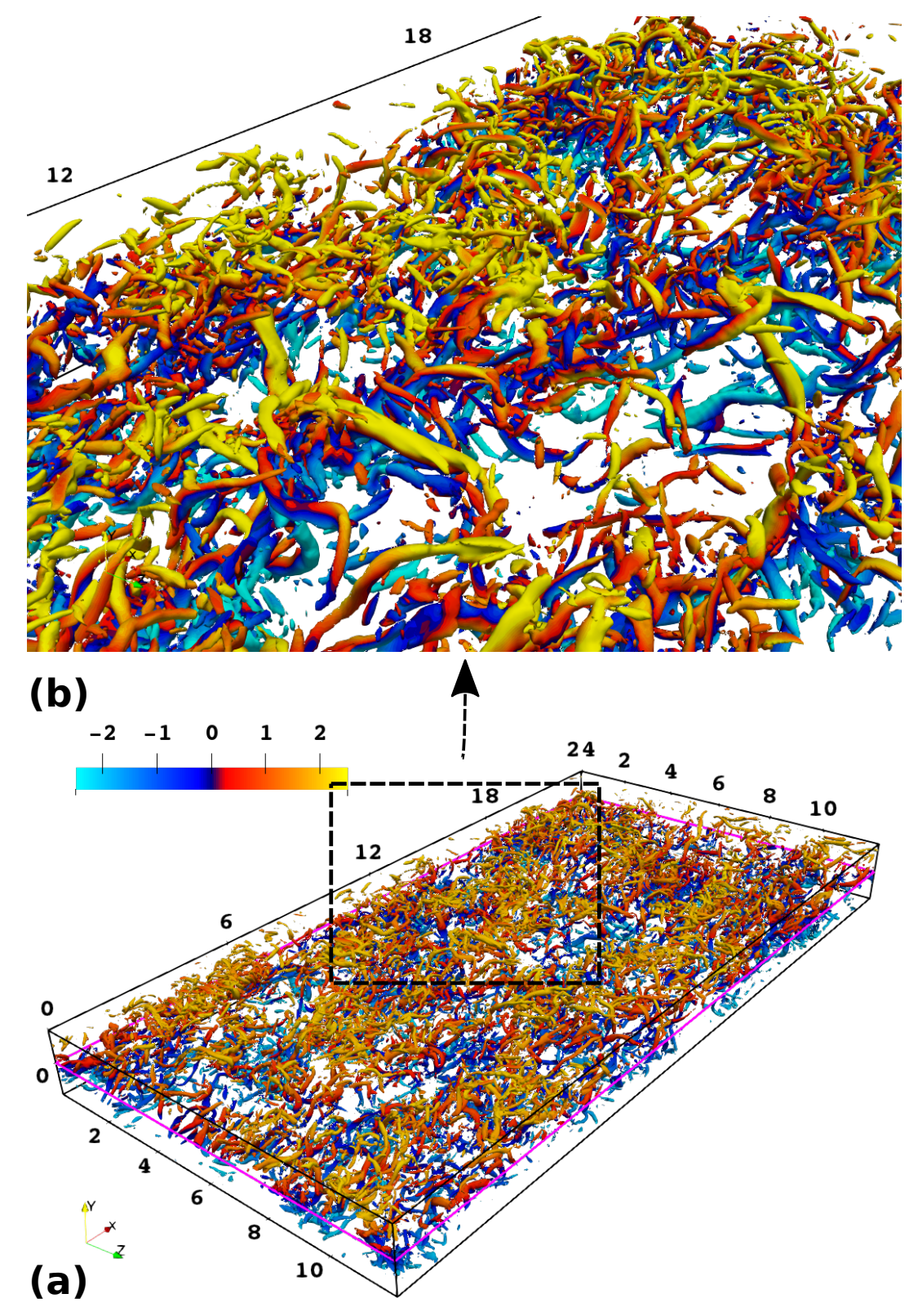

FIG. 4. (a): Iso-surfaces of the Q-criterion with an iso-value of $Q_{i s o}=200$ coloured by the magnitude of the streamwise velocity $(u)$ for the counter-flow case with $M=0.1, R e=400$ and a $24 \times 2 \times 12$ domain size at $t=1400$. (b): Zoomed view of the region within the dashed rectangle in (a). The legend limits are cut off for a better visualisation. The light violet lines in (a) show the outlines of the $x-z$ plane cutting through the channel centreline (the figure shows the full height of the channel from $y=-1$ to $y=1$ ).

power-law behaviour given by $\langle u\rangle=3|y|^{0.8}$.

As shown in figure 6, for the streamwise Reynolds stress $\left\langle u^{\prime} u^{\prime}\right\rangle$, all cases with different grid resolution show very similar profiles with comparable peak values. Comparing with 

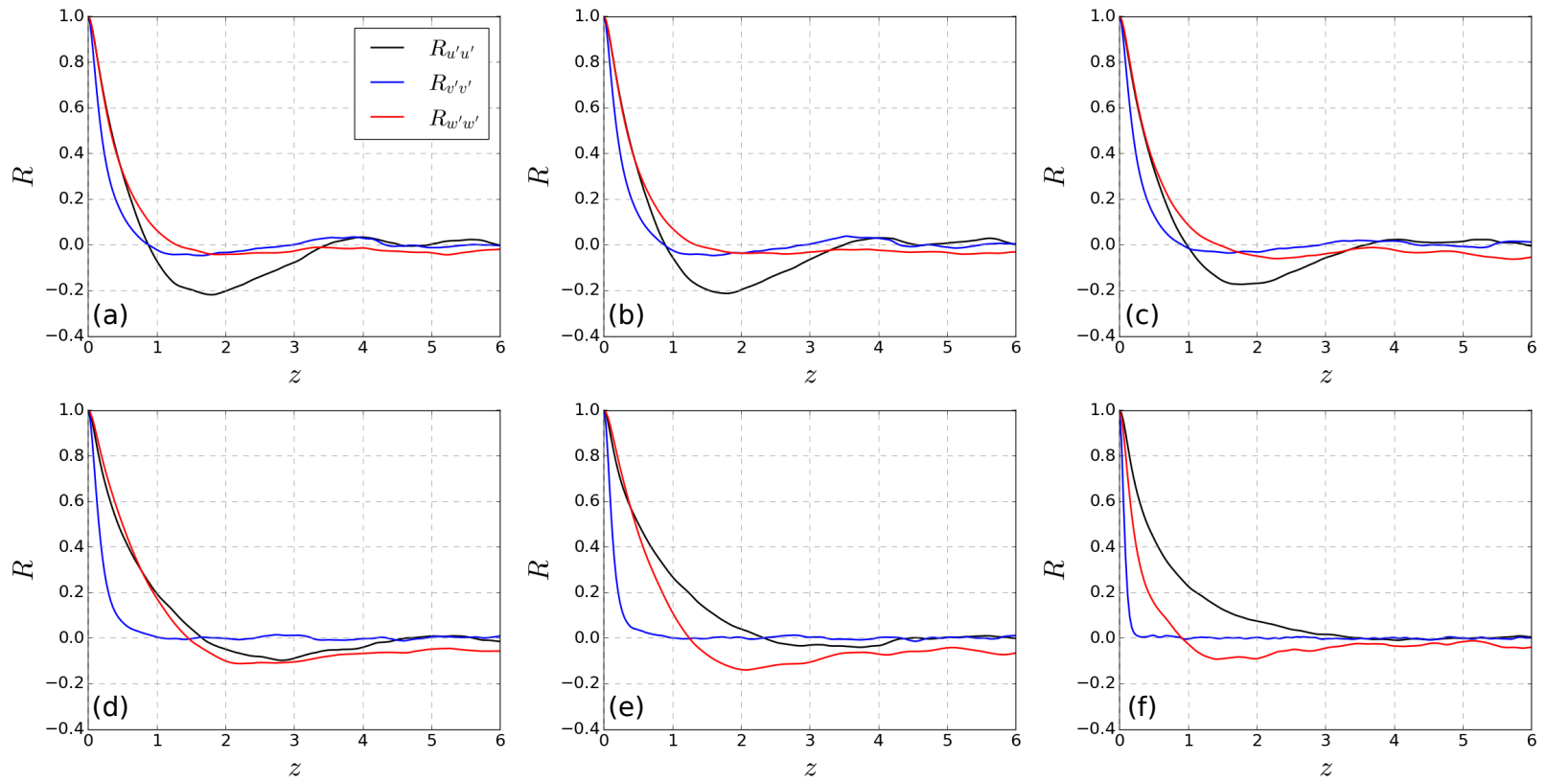

FIG. 5. The two-point correlations of $u^{\prime}, v^{\prime}$ and $w^{\prime}$ at different channel heights for the counter-flow case with a $24 \times 2 \times 12$ domain size. $(\mathrm{a}): y=0.0 ;(\mathrm{b}): y \approx 0.12 ;(\mathrm{c}): y \approx 0.35 ;(\mathrm{d}): y \approx 0.78 ;(\mathrm{e})$ : $y \approx 0.90 ;(\mathrm{f}): y \approx 0.997$ (first grid point above the wall).

the cases with larger domain sizes, the small-domain case exhibits around $4.0 \%$ higher and 11.0\% lower peak values of the wall-normal $\left\langle v^{\prime} v^{\prime}\right\rangle$ and the spanwise $\left\langle w^{\prime} w^{\prime}\right\rangle$ Reynolds stresses, respectively. However, $\left\langle u^{\prime} u^{\prime}\right\rangle$ is more dominant than the other two normal stresses, and the overall outcome is the very similar peak values of the turbulent Mach number, as shown in table I.

An interesting feature of figure 6 is the exactly linear behaviour of the shear stress and the approximately linear behaviour of the streamwise normal stress. As with conventional channel flow the linear variation of the shear stress derives from the imposed forcing. Neglecting the viscous terms, the momentum equation for such parallel mean flow reduces to:

$$
\frac{d}{d y}\langle\rho u v\rangle-\tanh (a y)=0
$$

Integrating equation 8 gives:

$$
\langle\rho u v\rangle-\frac{1}{a} \ln (|\cosh (a y)|)+C=0
$$

where the constant $C$ can be obtained by applying a no-slip boundary condition at the walls i.e. $\rho u v=0$ at $y= \pm 1$. For $a=10$ and $a=100$ the constant is $C \approx 0.930$ and $C \approx 0.993$, 

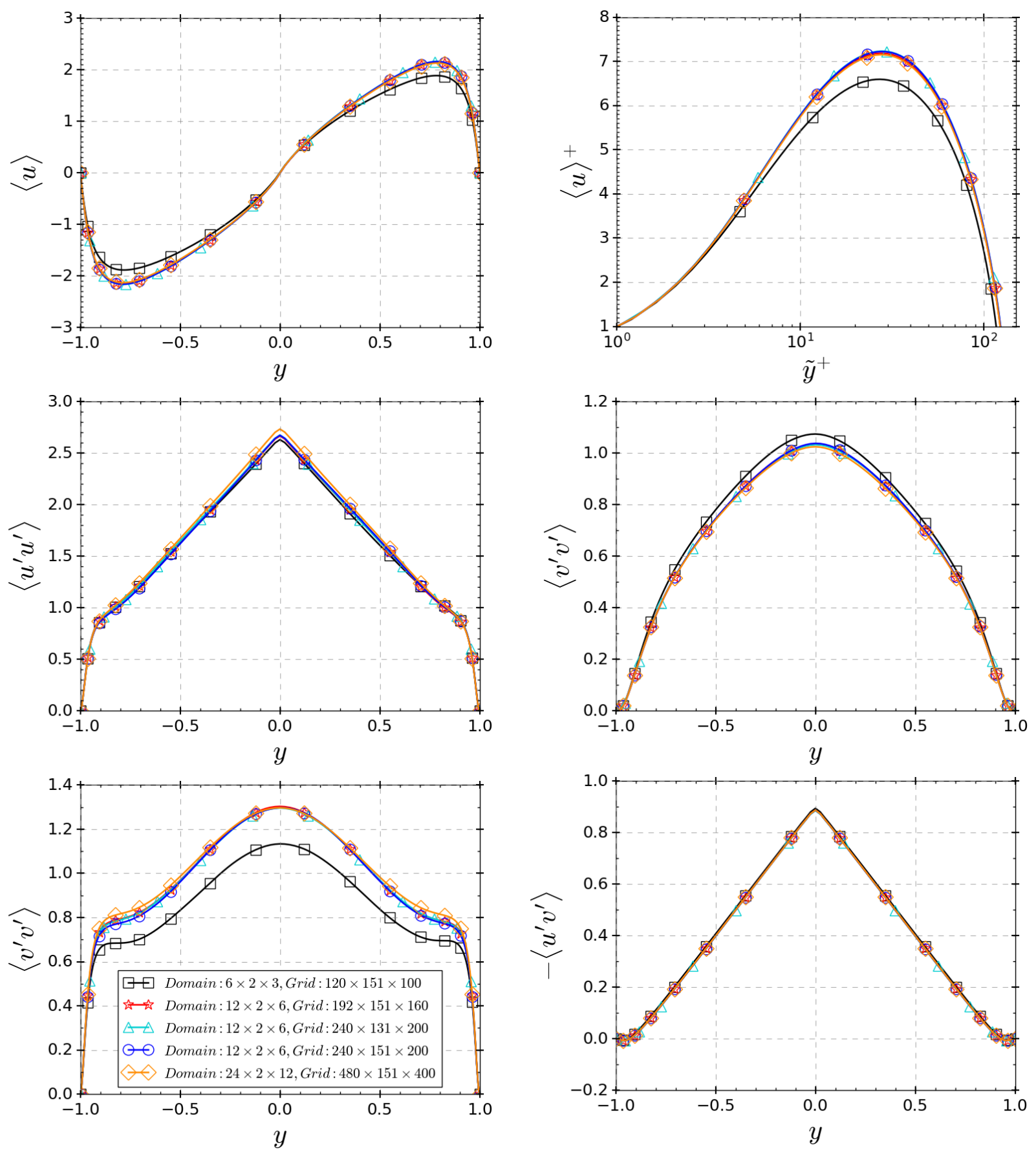

FIG. 6. Mean velocity and Reynolds stresses of the counter-flow cases with $M=0.1$ and $R e=400$ on different domain sizes and grid resolutions. Please note that for the $\langle u\rangle^{+}$sub-figure, the data is averaged over the two halves of the channel and $\tilde{y}^{+}$is relative to the wall. The markers are shown for every 10 grid points.

respectively. Equation 9 is plotted in figure 7 for different $a$ values along with $\langle\rho u v\rangle$ obtained by the present DNS for case 4 of table I $(M=0.1$ and $R e=400)$. The DNS has lower values 
compared to equation 9 due to the viscous effects close to the walls, which were neglected. The DNS case has a friction velocity of $u_{\tau} \approx 0.3$ as shown in table II which gives a wall shear stress of $\tau_{\text {wall }} \approx 0.1$. When this value is added to the value of $\langle\rho u v\rangle$ obtained by equation 9, the shifted curves can then be seen to match the DNS, also showing that the statistical averaging time is long enough to get well-converged statistics.

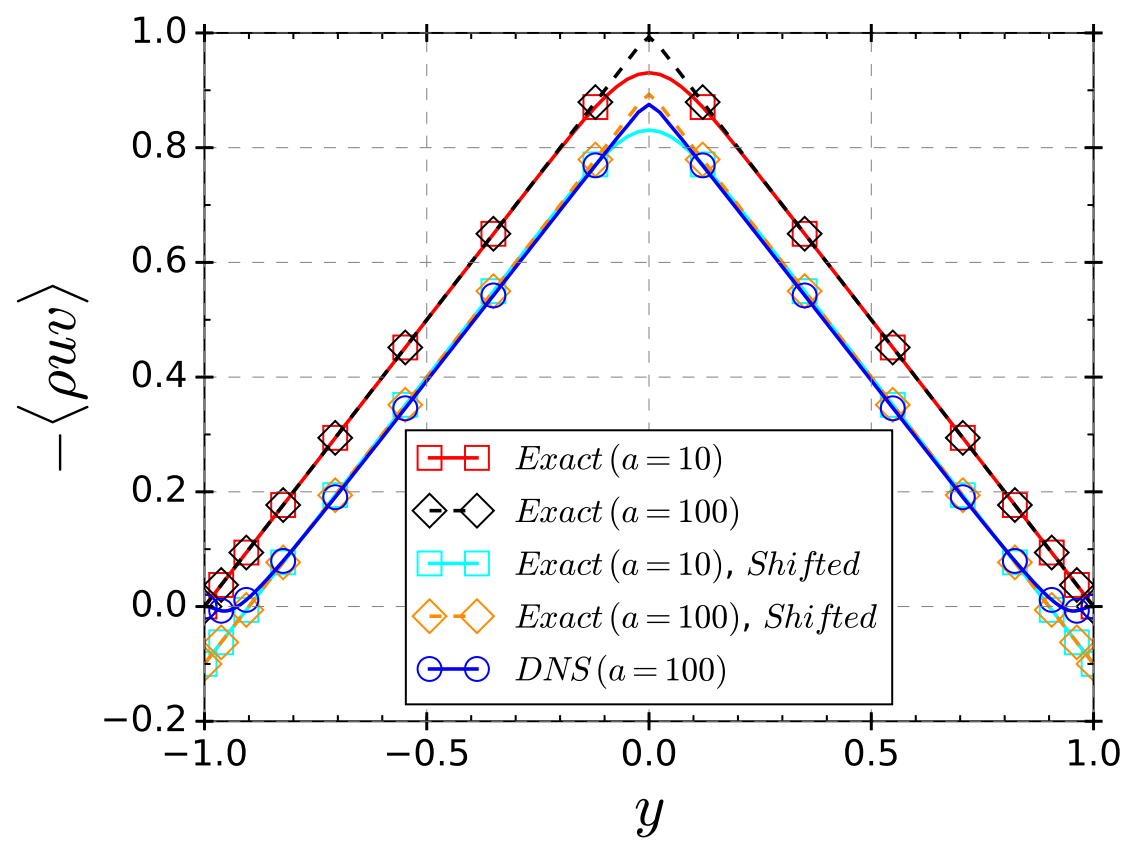

FIG. 7. A direct comparison between the exact solution of $\langle\rho u v\rangle$ based on equation 9 and the present DNS with $M=0.1$ and $R e=400$ (case 4 of table I). The markers are shown for every 10 grid points.

The approximate linearity of the streamwise normal stress $\left(\left\langle u^{\prime} u^{\prime}\right\rangle\right)$ motivated an investigation of the associated stress transport equation, which can be written as:

$$
0.0=\mathcal{P}_{11}-\epsilon_{11}+\Pi_{11}+\mathcal{D}_{11}^{t}+\mathcal{D}_{11}^{v}
$$

where, $\mathcal{P}_{11}, \epsilon_{11}, \Pi_{11}, \mathcal{D}_{11}^{t}$ and $\mathcal{D}_{11}^{v}$ are production, dissipation, pressure strain, turbulent diffusion and viscous diffusion terms, respectively, defined as:

$$
\begin{gathered}
\mathcal{P}_{11}=-2\left\langle u^{\prime} v^{\prime}\right\rangle \frac{\partial\langle u\rangle}{\partial y}, \\
\epsilon_{11}=2\left\langle\frac{d u^{\prime}}{d x} \tau_{11}^{\prime}+\frac{d u^{\prime}}{d y} \tau_{12}^{\prime}+\frac{d u^{\prime}}{d z} \tau_{13}^{\prime}\right\rangle,
\end{gathered}
$$




$$
\begin{gathered}
\Pi_{11}=2\left\langle p^{\prime} \frac{d u^{\prime}}{d x}\right\rangle, \\
\mathcal{D}_{11}^{t}=-\frac{d\left\langle u^{\prime} u^{\prime} v^{\prime}\right\rangle}{d y}, \\
\mathcal{D}_{11}^{v}=\frac{d}{d y}\left(\frac{1}{R e} \frac{d\left\langle u^{\prime} u^{\prime}\right\rangle}{d y}\right) .
\end{gathered}
$$

The above budget terms are plotted in figure 8 for the counter-flow cases with $6 \times 2 \times 3$ and $12 \times 2 \times 6$ domain sizes $(M=0.1$ and $R e=400)$. The budget terms for a steady parallel mean flow sum up to zero as demonstrated by the present DNS. It can also be seen in figure 8 that increasing the domain dimensions does not have a significant influence on the budget terms. The peak of the production term is at the channel centreline, which is consistent with the profiles of the Reynolds stresses as shown in figure 6. The dissipation term is approximately constant across the channel core, while the diffusion terms are only of consequence near the walls. The flow is clearly far from an equilibrium state between production and dissipation, with the dissipation only the fourth largest term near the centreline, where the main balance to the production comes from the turbulent diffusion and the pressure strain term. From the perspective of the stress transport equation, the linearity of the normal stress seems to be due to a complicated balance in the spatial distribution of the production, pressure strain and turbulent diffusion terms which change rapidly in the wall-normal direction.

\section{EFFECT OF THE REYNOLDS NUMBER}

\section{A. Mean flow and turbulence statistics}

Figure 9 shows a direct comparison between the cases with a $6 \times 2 \times 3$ domain size, $M=0.1$ and $R e=400,800$ and 1600 (cases 1, 7 and 8 of table I) based on various mean flow quantities, including the streamwise velocity $\langle u\rangle$, temperature $\langle T\rangle$, density $\langle\rho\rangle$ and Mach number $\langle M\rangle$, and also the turbulent Mach number $M_{t}$. Increasing Re does not significantly affect the mean flow. For instance, the peak mean streamwise velocity increases by around $9.8 \%$ and $12.7 \%$ when $R e$ increases to 800 and 1600, respectively. Moreover, the peak turbulent Mach number only increases by around $7.7 \%$ and $8.8 \%$ when $R e$ increases to 800 and 1600, respectively. When plotted in wall units it can be seen that the viscous sub-layer extends outwards, but at the highest $R e$ the peak $\langle u\rangle^{+}$remains under 10 , increasing by 1.5 

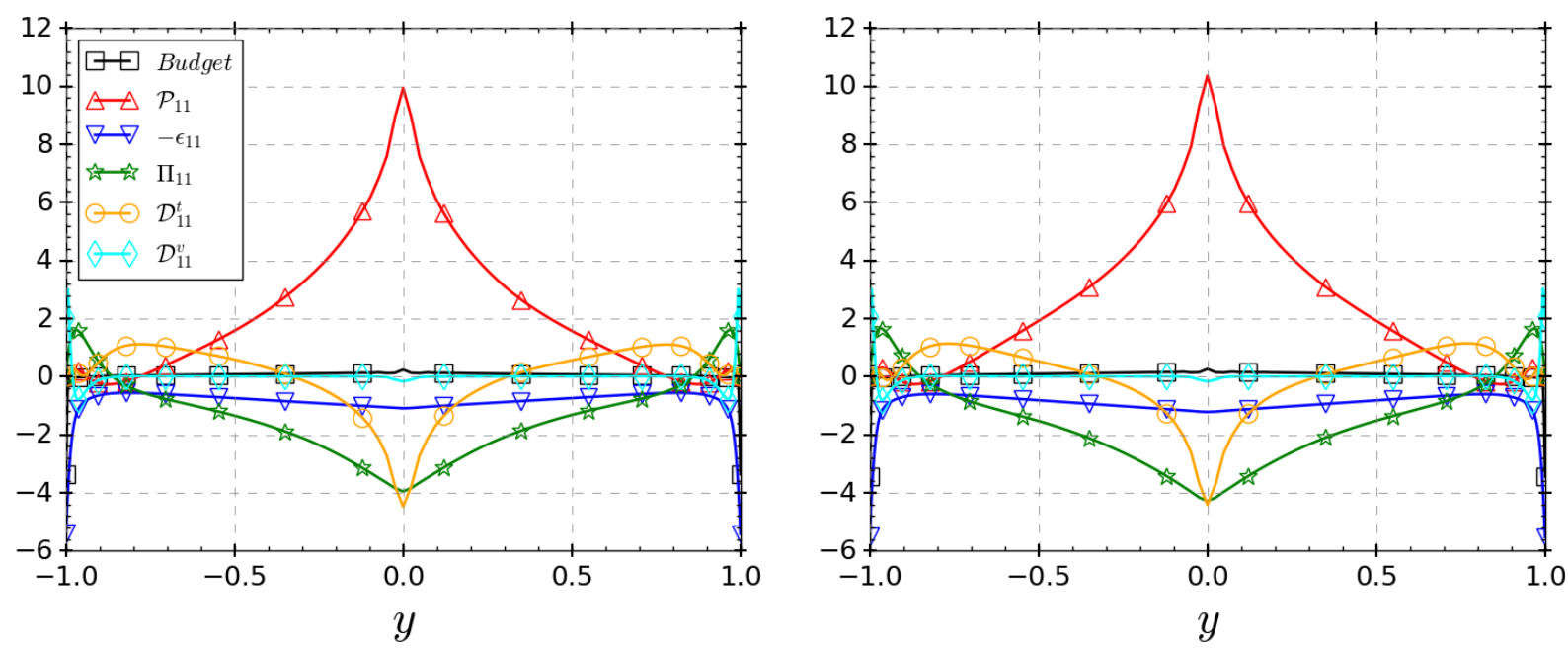

FIG. 8. Energy budget of the streamwise normal stress $\left\langle u^{\prime} u^{\prime}\right\rangle$ and its terms for the cases with $M=0.1, R e=400$ and left: domain size of $6 \times 2 \times 3$ and right: domain size of $12 \times 2 \times 6$. The markers are shown for every 10 grid points.

for each doubling of $R e$. The mean temperature is seen to be more sensitive than the mean density, indicating that the mean pressure is increasing as the Reynolds number increases. From the wall-normal momentum equation this would equate to a change in the wall-normal turbulent stress, as seen in Figure 10, which provides the Reynolds stresses. However, the dependency of the mean temperature to the Reynolds number is quite small and the peak mean temperature at the channel centreline increases by $\sim 3.5 \%$ as the Reynolds number is doubled. On the other hand, the dependency of the mean density to the Reynolds is much lower (almost not noticeable) compared to that of the mean temperature. All the normal stresses exhibit more sensitivity to $R e$ and their peak values noticeably increase as the $R e$ increases, with values shown in table III. Both the normal stresses based on wall-parallel fluctuations show the development of a near-wall peak that indicates the emergence of a separate mechanism of near-wall turbulence production which will be studied further in the next subsection. The shear stress is again linear, in accordance with the imposed forcing, with a slight increase in the centreline shear stress as Re increases.

\section{B. Flow structure}

To complement the statistical picture obtained in the previous subsection, it is also useful to consider some instantaneous flowfields. Figure 11 shows the instantaneous streamwise 

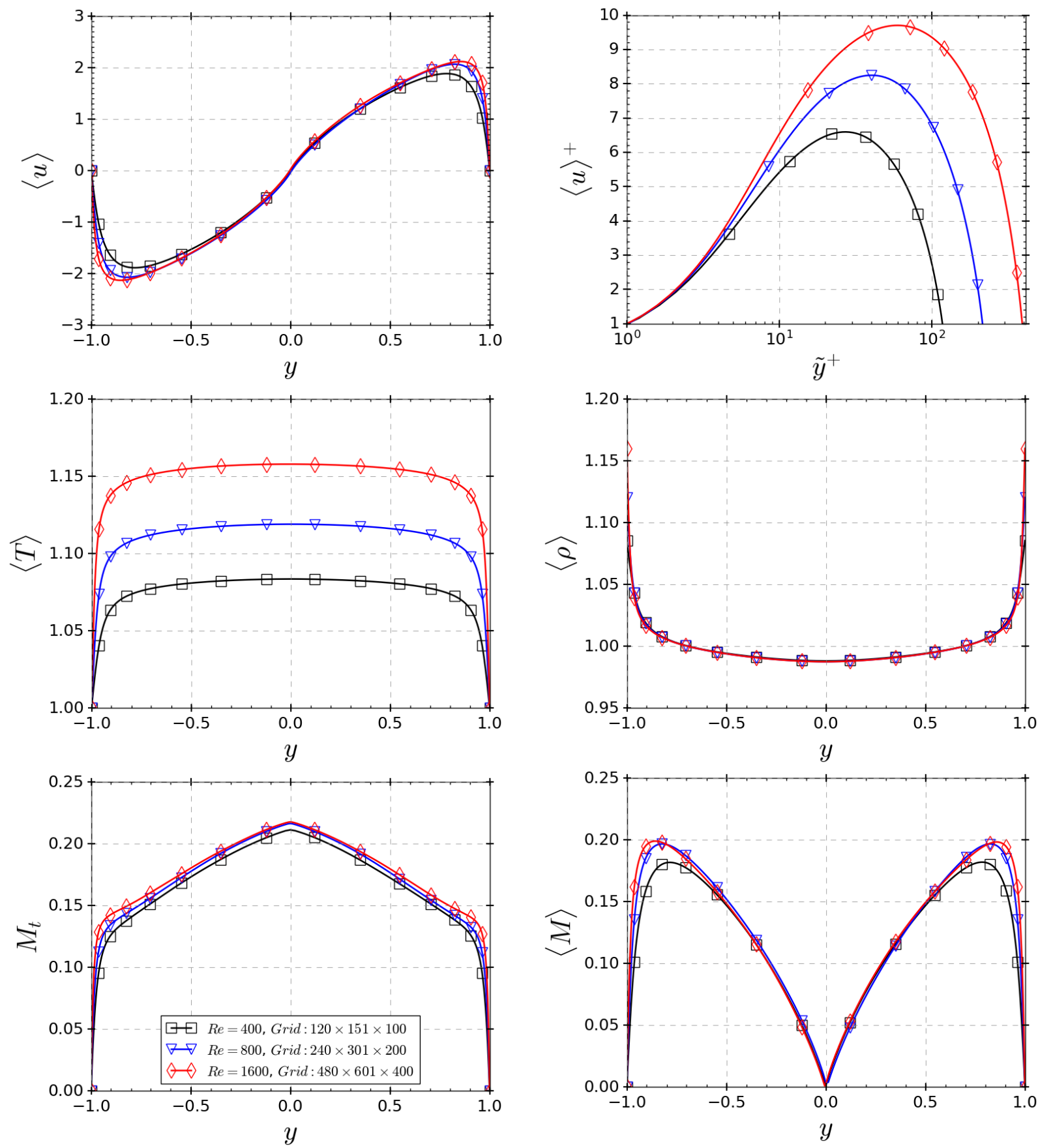

FIG. 9. Mean velocity, temperature, density and Mach number profiles and the turbulent Mach number $\left(M_{t}\right)$ profile of the counter-flow cases with $M=0.1$ and $R e=400,800$ and 1600. Please note that for the $\langle u\rangle^{+}$sub-figure, the data is averaged over the two halves of the channel and $\tilde{y}^{+}$is relative to the wall. The markers are shown for every 10, 20 and 40 grid points for the cases with $R e=400,800$ and 1600, respectively.

velocity on $x-z$ planes at different channel heights. At the lowest $R e$, the flow near the wall 

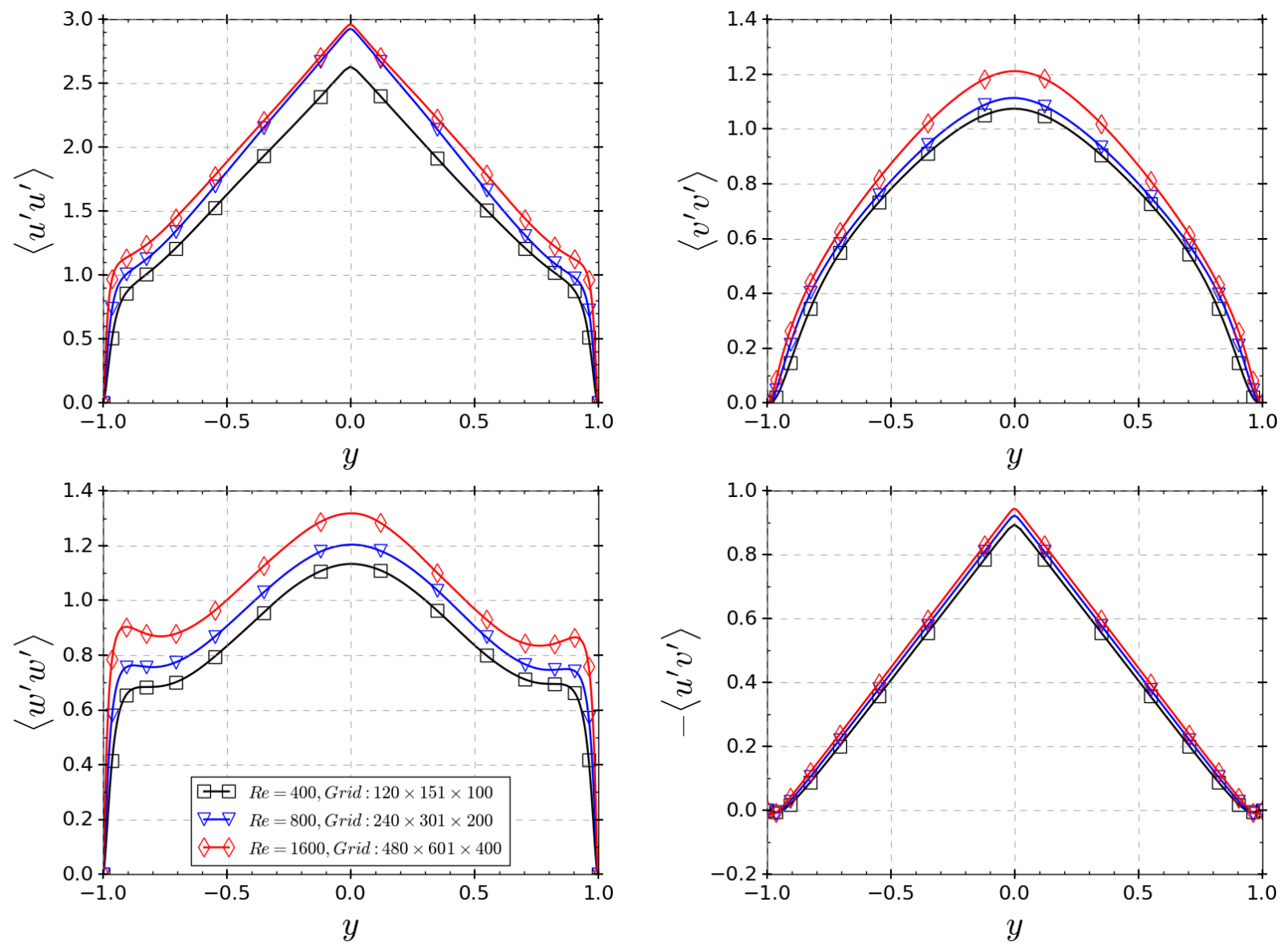

FIG. 10. Reynolds stresses for the counter-flow cases with $M=0.1$ and $R e=400,800$ and 1600 . The markers are shown for every 10, 20 and 40 grid points for the cases with $R e=400,800$ and 1600 , respectively.

TABLE III. Peak values of the Reynolds stresses of the cases with $R e=400,800$ and 1600 .

\begin{tabular}{cccccc}
\hline Case & $R e$ & $\left\langle u^{\prime} u^{\prime}\right\rangle_{p}$ & $\left\langle v^{\prime} v^{\prime}\right\rangle_{p}$ & $\left\langle w^{\prime} w^{\prime}\right\rangle_{p}$ & $-\left\langle u^{\prime} v^{\prime}\right\rangle_{p}$ \\
\hline 1 & 400 & 2.630 & 1.074 & 1.133 & 0.894 \\
7 & 800 & 2.927 & 1.113 & 1.203 & 0.922 \\
8 & 1600 & 2.959 & 1.211 & 1.317 & 0.943 \\
\hline
\end{tabular}

is buffeted by the turbulence originating at the centreline. In particular it is noteworthy that significant regions of reverse flow are present near the wall. This is something that develops in boundary-layer flows at high enough $R e$, as the turbulence intensity in the log-law region of the boundary layer increases above that of the self-sustaining near-wall turbulence. The present configuration contains this phenomenon even at low $R e$. 
Additional smaller-scale streaky structures are visible at $\tilde{y}^{+}=12$ for the case with $R e=$ 1600 (and less evidently on the same plane height for the case with $R e=800$ ). These streaks are superimposed on the large-scale unsteadiness, but are of a scale that suggests they are connected to the usual streaks seen at the edge of the viscous sublayer in turbulent channel and boundary layer flows. For reference, given that $R e_{\tau}$ is approximately 400, the near-wall streak width in the $y$ direction would be $\delta y=0.25$ in the channel scale shown in figure 11. The additional turbulence intensity from these features would explain the increases in $\left\langle u^{\prime} u^{\prime}\right\rangle$ and $\left\langle w^{\prime} w^{\prime}\right\rangle$ seen in Figure 10. It was previously noted that the peak $\langle u\rangle^{+}$is just under 10, with the peak at $\tilde{y}^{+}=60$. This is just below what would be required to sustain a near-wall turbulence cycle, so it seems likely that the streak here form as a response to the outer-layer forcing. Additional evidence for this is that the streaks that are visible in figure 11 clearly align with the imposed unsteady flow, rather than being strictly aligned with the $x$ axis. Nevertheless, it appears that one would not have to go much higher in $R e$ (perhaps one more doubling) before a self-sustaining near-wall turbulence cycle would be present, giving an additional means of turbulence production on top of the main inflectional shear layer.

(a)
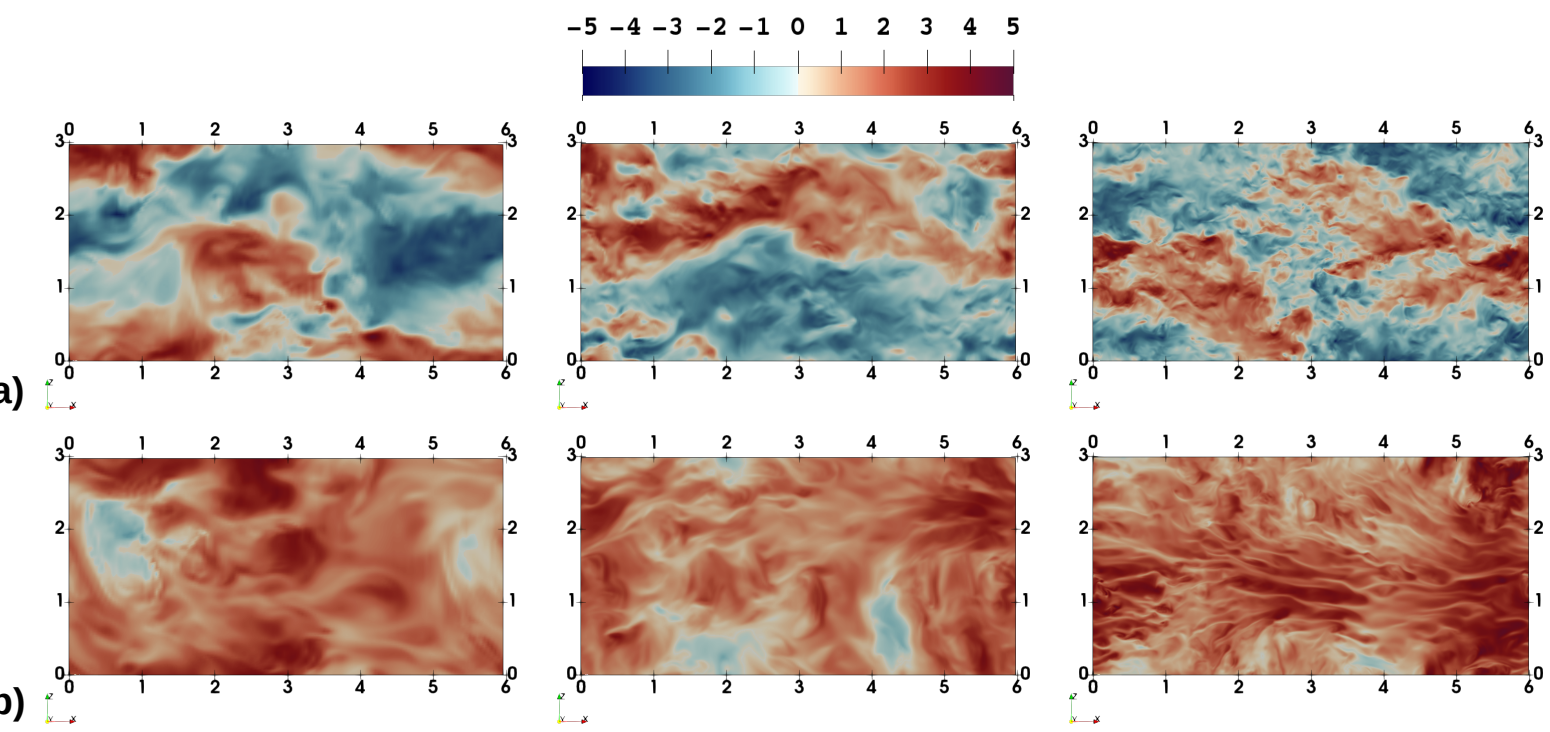

FIG. 11. Instantaneous field of the streamwise velocity $(u)$ on $x-z$ planes at different channel heights for the counter-flow cases with $M=0.1$ and $R e=400$ (left), $R e=800$ (middle) and $R e=1600$ (right) at $t=400$. (a): $y=0.0$ (channel centre) and (b): $\tilde{y}^{+}=12$. 


\section{EFFECT OF THE MACH NUMBER}

Figure 12 shows a direct comparison between the cases with a $12 \times 2 \times 6$ domain size and $M=0.1$ and 0.4 (cases 4 and 6 of table I) based on various mean flow quantities, including the streamwise velocity $\{u\}$, temperature $\langle T\rangle$, density $\langle\rho\rangle$ and Mach number $\langle M\rangle$, and also the turbulent Mach number $M_{t}$. Additionally, figure 13 provides the Favre Reynolds stresses of the same counter-flow cases. As shown in table II, when $M$ increases from 0.1 to 0.4 the friction Reynolds number, $R e_{\tau}$, increases by around 47.0\%. Also, the friction velocity, $u_{\tau}$, reduces by around $29.7 \%$, and the peak mean normalised velocity increases by $34.8 \%$. The flow significantly heats up when $M$ increases, for instance the peak mean temperature is around 2.3 times higher for the case with $M=0.4$ compared to the case with $M=0.1$. Mean and turbulent Mach numbers also show significant increases when $M$ increases. The peak turbulent Mach number increases from 0.21 to 0.6 when $M$ increases from 0.1 to 0.4. With respect to the normal stresses, as shown in figure 13, by increasing the Mach number, the peak streamwise stress increases and the peaks of the other two normal stresses reduce. For instance, as shown in table IV, the peak $\langle\rho\rangle\left\{u^{\prime \prime} u^{\prime \prime}\right\}$ increases by around $0.5 \%$ when $M$ increases from 0.1 to 0.4 . The Favre shear stress $\langle\rho\rangle\left\{u^{\prime \prime} v^{\prime \prime}\right\}$ remains almost intact when the Mach number increase and varies linearly with $y$ as also seen in the previous sections.

Figure 14 shows the vorticity fluctuation $\left(\omega^{\prime}=\sqrt{\left\langle\omega_{x}^{2}+\omega_{y}^{2}+\omega_{z}^{2}\right\rangle}\right)$ and the components of the vorticity turbulent fluctuations for the cases with different Mach numbers. By increasing the Mach number, values of the vorticity fluctuation and the vorticity turbulent fluctuations reduce noticeably. This trend is comparable to the relationship between the spanwise and wall-normal Reynolds stresses and the Mach number as seen in figure 13. The vorticity reduction is a sign for the reduction of three-dimensional structures in the flow and hence turbulence stabilisation.

In a conventional plane channel flow, Coleman et al [20] were able to reach turbulent Mach numbers around 0.35 for which the changes relative to incompressible flow were explainable based on the mean density changes. The higher fluctuating Mach number found in the counter-flow channel proposed in the present study suggests more scope for exploring true compressibility effects. Indeed, a further increase in the Mach number to $M=0.7$ was found to give turbulent flow containing eddy shocklets with a turbulent Mach number beyond $M_{t}=0.9$. This then necessitates a change to a shock-capturing numerical method requiring additional verifications, hence being better deferred to a future publication. Mild 

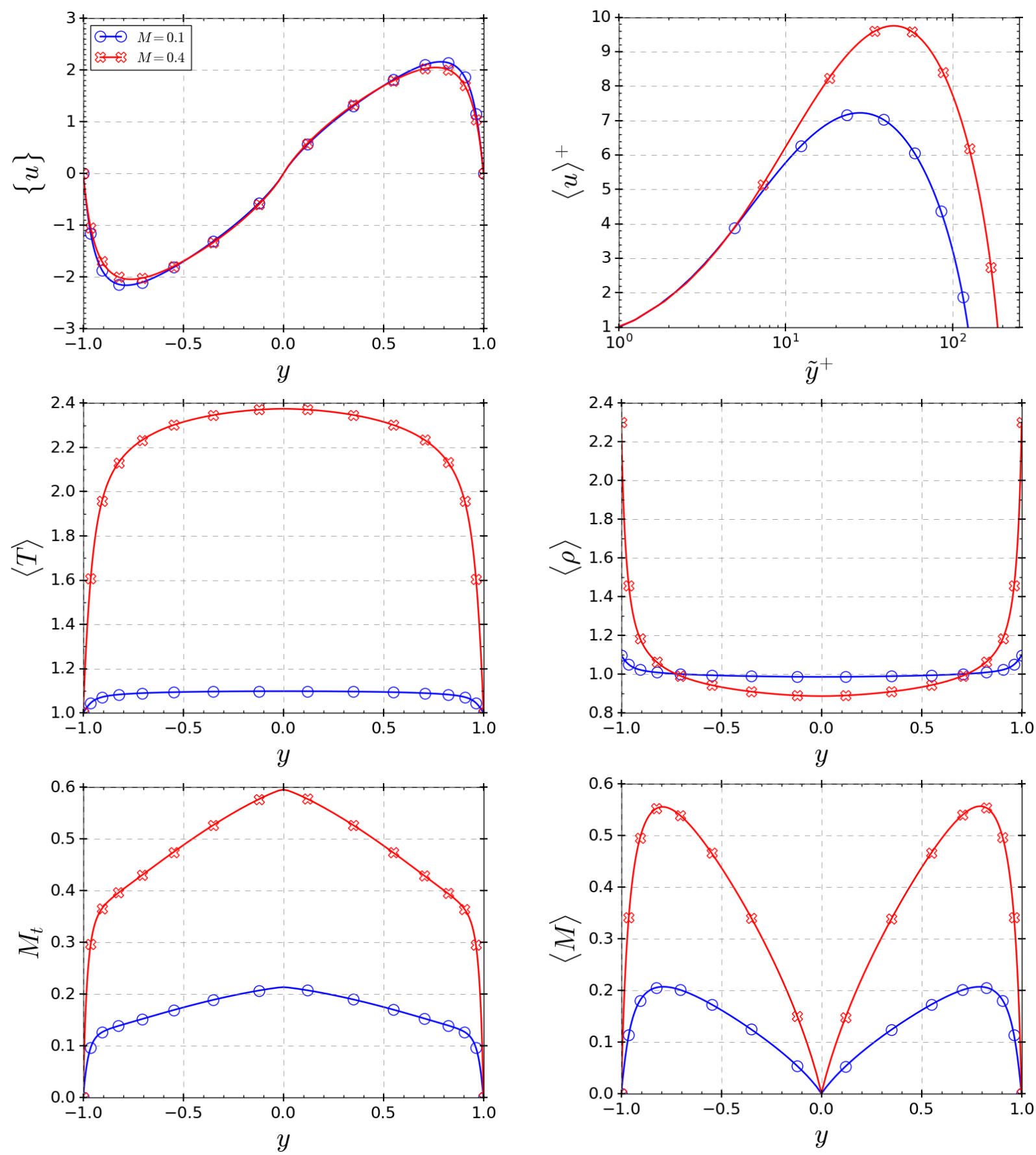

FIG. 12. Mean velocity, temperature, density and Mach number profiles and the turbulent Mach number $\left(M_{t}\right)$ profile of the counter-flow cases with $M=0.1$ and $0.4(R e=400)$. Please note that for the $\langle u\rangle^{+}$sub-figure, the data is averaged over the two halves of the channel and $\tilde{y}^{+}$is relative to the wall. The markers are shown for every 10 grid points.

compressibility effects are observed up to $M=0.4$ which can be quantified using the dilatation defined as $\theta=\frac{\partial u}{\partial x}+\frac{\partial v}{\partial y}+\frac{\partial w}{\partial z}$. Specifically, negative values of the dilatation (below a 

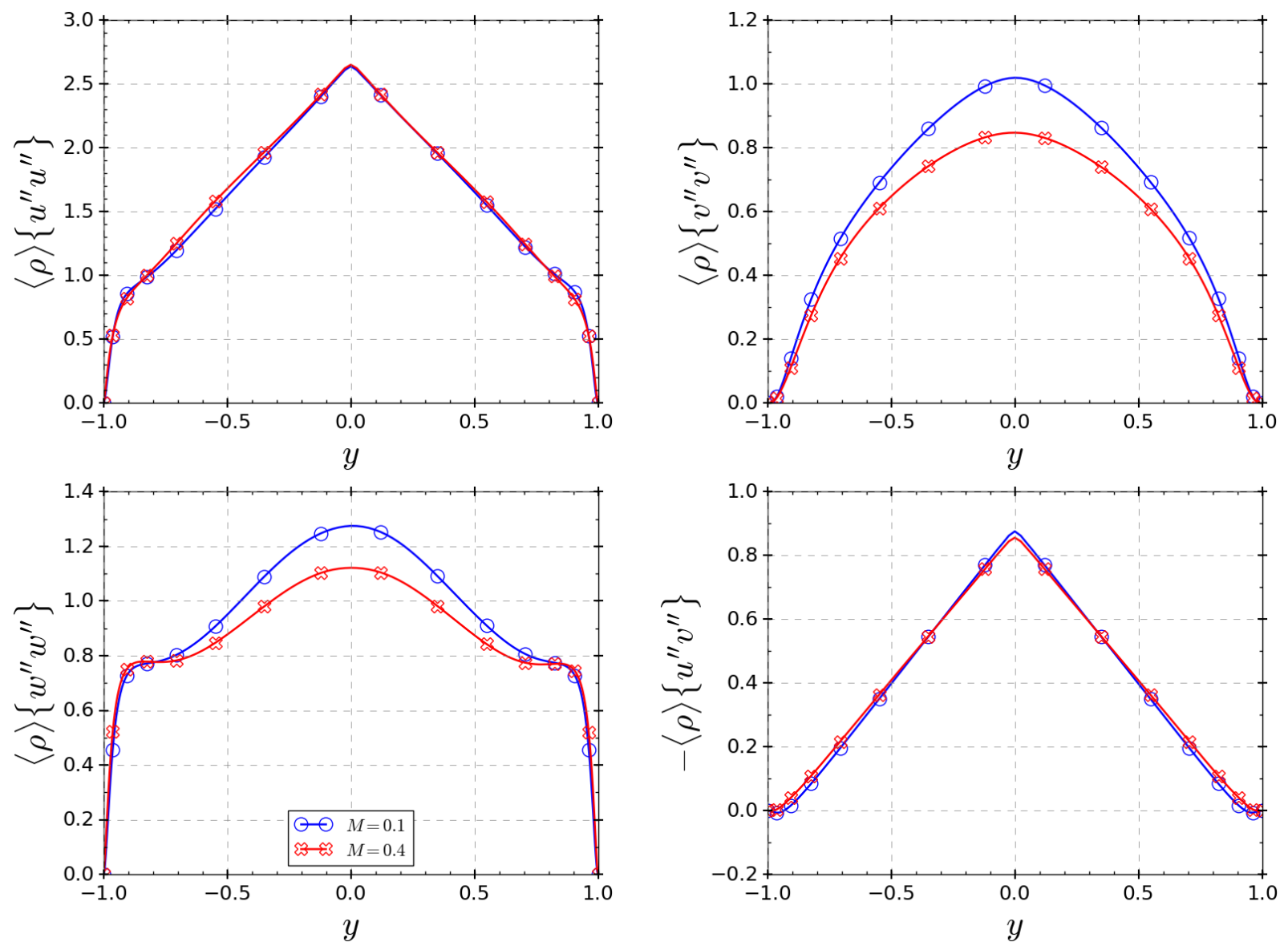

FIG. 13. Favre Reynolds stresses for the counter-flow cases with $M=0.1$ and $0.4(R e=400)$. The markers are shown for every 10 grid points.

certain threshold) can be associated with the existence of compression waves and/or shocklets $[37,38]$. Figure 15 shows the Probability Density Function (PDF) of the dilatation $(\theta)$ on a $x-z$ plane at $y \approx 0.51$ for the counter-flow cases with $M=0.1$ and 0.4 . It is observed that the dilatation trend widens when moving from $M=0.1$ to $M=0.4$ but stays almost symmetric indicating that shocklets are not detected in the flow.

TABLE IV. Peak values of the Favre stresses of the counter-flow cases with $R e=400$ and $M=0.1$ and 0.4 .

\begin{tabular}{cccccc}
\hline Case & $M$ & $\langle\rho\rangle\left\{u^{\prime \prime} u^{\prime \prime}\right\}_{p}$ & $\langle\rho\rangle\left\{v^{\prime \prime} v^{\prime \prime}\right\}_{p}$ & $\langle\rho\rangle\left\{w^{\prime \prime} w^{\prime \prime}\right\}_{p}$ & $-\langle\rho\rangle\left\{u^{\prime \prime} v^{\prime \prime}\right\}_{p}$ \\
\hline 4 & 0.1 & 2.638 & 1.019 & 1.274 & 0.875 \\
6 & 0.4 & 2.650 & 0.847 & 1.121 & 0.855 \\
\hline
\end{tabular}



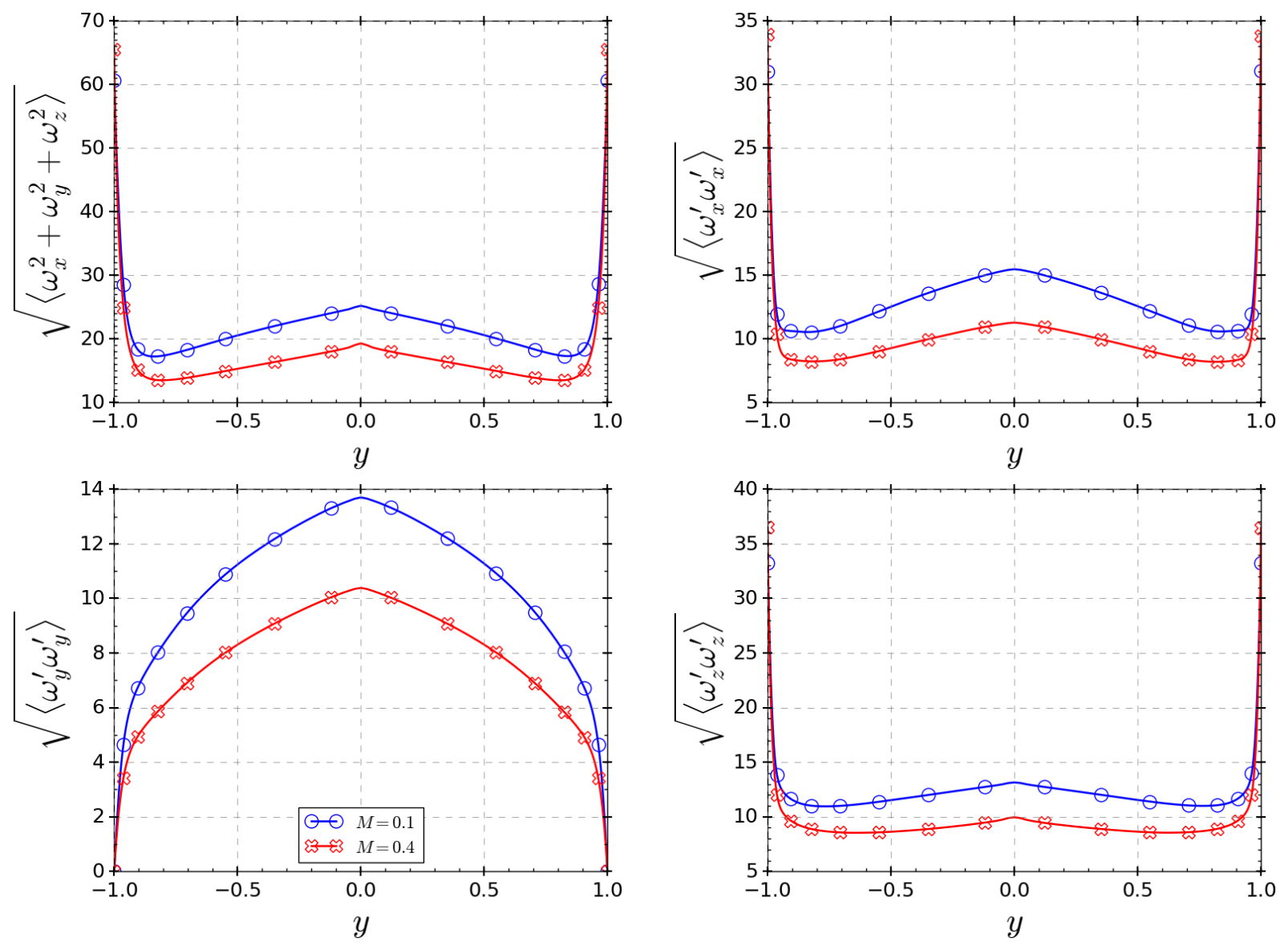

FIG. 14. Vorticity fluctuation and the components of the vorticity turbulent fluctuations of the counter-flow cases with $M=0.1$ and $0.4(R e=400)$. The markers are shown for every 10 grid points.

\section{CONCLUSIONS}

A counter-flow turbulent channel configuration, amenable to simulation and modelling, has been introduced. The counter-flow channel has periodic streamwise and spanwise boundaries and isothermal no-slip walls and is driven by a mean pressure gradient introduced by adding a forcing term to the streamwise momentum and total energy equations. The forcing term was a tangent hyperbolic function that forced the flow in opposite directions on the upper and lower halves of the channel. Direct numerical simulations of the counter-flow configuration were performed for combinations of various domains sizes $(6 \times 2 \times 3,12 \times 2 \times 6$ and $24 \times 2 \times 12)$, grid resolutions, friction Mach numbers $(M=0.1$ and 0.4$)$ and Reynolds numbers $(R e=400,800$ and 1600). The OpenSBLI framework, a high-order finite difference automatic code generation computational fluid dynamics framework, was used to perform 


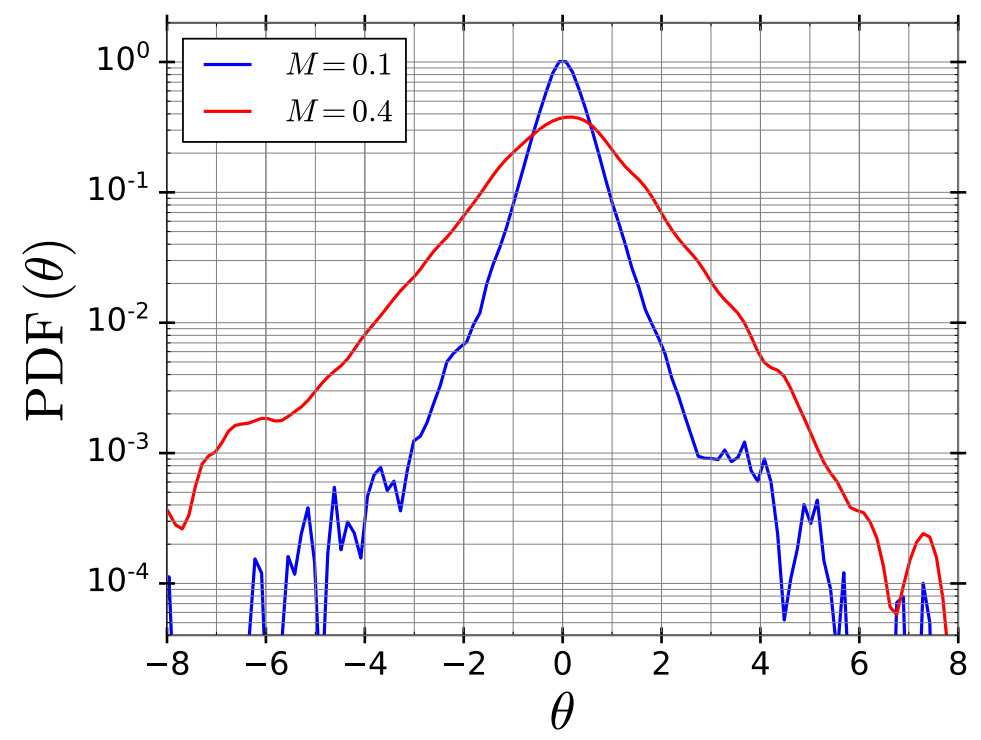

FIG. 15. Probability density of the dilatation on a $x-z$ plane at $y \approx 0.51$ and $t=1400$ for the counter-flow cases with $M=0.1$ and $0.4(R e=400)$.

the high-fidelity simulations.

The counter-flow results showed a linear shear-stress behaviour consistent with the forcing and also an approximately linear streamwise normal stress behaviour over $75 \%$ of the channel width. In some sense this mimics high Reynolds turbulent boundary layers giving reverse flow near the wall, with near-wall streaks aligned with disturbances further away from the wall. As the Reynolds number increased, the near-wall streaks became more pronounced, leading to local peaks in the streamwise and spanwise normal stresses. With an increase of the Mach number up to $M=0.4$, it was shown that turbulent Mach numbers up to 0.6 could be obtained. Thus the counter-flow configuration seems to provide a useful framework to study some of the fundamental physics associated with high Reynolds number and high Mach number turbulent flows, as well as being a model problem exhibiting a high degree of sustained turbulent mixing.

The DNS counter-flow channel results discussed in this paper are openly available in Zenodo at https://doi.org/10.5281/zenodo.4635349.

\section{ACKNOWLEDGMENTS}

Dr Arash Hamzehloo is funded by the UK Turbulence Consortium (UKTC) under the EPSRC grant EP/R029326/1. Dr David J Lusher was funded by an EPSRC Centre for Doc- 
toral Training grant (EP/L015382/1). The authors acknowledge the use of the Cambridge Tier-2 system operated by the University of Cambridge Research Computing Service under an EPSRC Tier-2 capital grant (EP/P020259/1) to perform simulations reported here. The OpenSBLI code is available at https://opensbli.github.io.

[1] J. Humphrey and S. Li, Tilting, stretching, pairing and collapse of vortex structures in confined counter-current flow, Journal of Fluids Engineering 103 (1981).

[2] P. J. Strykowski and R. Wilcoxon, Mixing enhancement due to global oscillations in jets with annular counterflow, AIAA Journal 31, 564 (1993).

[3] D. J. Forliti, B. A. Tang, and P. J. Strykowski, An experimental investigation of planar countercurrent turbulent shear layers, Journal of Fluid Mechanics 530, 241 (2005).

[4] J. A. Humphrey, J. L. Rosales, L. A. Legendre, J. P. LeDuc, and J. P. Landers, Vortex dynamics in confined counter-current shearing flows with applications to mixing, International Journal of Heat and Fluid Flow 29, 1089 (2008).

[5] P. J. Strykowski, A. Krothapalli, and D. Forliti, Counterflow thrust vectoring of supersonic jets, AIAA Journal 34, 2306 (1996).

[6] F. Alvi and P. Strykowski, Forward flight effects on counterflow thrust vector control of a supersonic jet, AIAA Journal 37, 279 (1999).

[7] R. Gillgrist, D. Forliti, and P. J. Strykowski, On the mechanisms affecting fluidic vectoring using suction, Journal of Fluids Engineering 129 (2007).

[8] S. Lonnes, D. Hofeldt, and P. Strykowski, Flame speed control using a countercurrent swirl combustor, in 36th AIAA Aerospace Sciences Meeting and Exhibit (1998) p. 352.

[9] P. Strykowski, D. Forliti, and R. Gillgrist, Controlling flame speed using countercurrent shear, in Proceedings of 1999 IEEE International Conference on Control Applications (1999).

[10] P. Huerre and P. A. Monkewitz, Absolute and convective instabilities in free shear layers, Journal of Fluid Mechanics 159, 151 (1985).

[11] P. J. Strykowski, D. Niccum, et al., The stability of countercurrent mixing layers in circular jets, Journal of Fluid Mechanics 227, 309 (1991).

[12] S. Lee, S. K. Lele, and P. Moin, Eddy shocklets in decaying compressible turbulence, Physics of Fluids A: Fluid Dynamics 3, 657 (1991).

[13] S. Sarkar, The stabilizing effect of compressibility in turbulent shear flow, Journal of Fluid 
Mechanics 282, 163 (1995).

[14] S. K. Lele, Compressibility effects on turbulence, Annual Review of Fluid Mechanics 26, 211 (1994).

[15] J. B. Freund, S. K. Lele, and P. Moin, Compressibility effects in a turbulent annular mixing layer. Part 1. Turbulence and growth rate, Journal of Fluid Mechanics 421, 229 (2000).

[16] J. Yao and F. Hussain, Turbulence statistics and coherent structures in compressible channel flow, Physical Review Fluids 5, 084603 (2020).

[17] M. R. Mankbadi, J. R. DeBonis, and N. J. Georgiadis, Large-eddy simulation of a compressible mixing layer and the significance of inflow turbulence, in 55th AIAA Aerospace Sciences Meeting (2017) p. 0316.

[18] A. W. Vreman, N. Sandham, and K. Luo, Compressible mixing layer growth rate and turbulence characteristics, Journal of Fluid Mechanics 320, 235 (1996).

[19] J. Kim, P. Moin, and R. Moser, Turbulence statistics in fully developed channel flow at low Reynolds number, Journal of Fluid Mechanics 177, 133 (1987).

[20] G. Coleman, J. Kim, and R. Moser, A numerical study of turbulent supersonic isothermal-wall channel flow, Journal of Fluid Mechanics 305, 159 (1995).

[21] R. Johnstone, G. N. Coleman, and P. R. Spalart, The resilience of the logarithmic law to pressure gradients: evidence from direct numerical simulation, Journal of Fluid Mechanics 643, $163(2010)$.

[22] F. Waleffe, On the three-dimensional instability of strained vortices, Physics of Fluids A: Fluid Dynamics 2, 76 (1990).

[23] V. Mantič-Lugo and F. Gallaire, Self-consistent model for the saturation mechanism of the response to harmonic forcing in the backward-facing step flow, Journal of Fluid Mechanics 793, 777 (2016).

[24] V. Mantič-Lugo and F. Gallaire, Saturation of the response to stochastic forcing in twodimensional backward-facing step flow: A self-consistent approximation, Physical Review Fluids 1, 083602 (2016).

[25] A. Hamzehloo, D. J. Lusher, S. Laizet, and N. D. Sandham, On the performance of WENO/TENO schemes to resolve turbulence in DNS/LES of high-speed compressible flows, International Journal for Numerical Methods in Fluids 93, 176 (2021).

[26] F. Ducros, F. Laporte, T. Soulères, V. Guinot, P. Moinat, and B. Caruelle, High-order fluxes for conservative skew-symmetric-like schemes in structured meshes: application to compress- 
ible flows, Journal of Computational Physics 161, 114 (2000).

[27] N. D. Sandham, Q. Li, and H. C. Yee, Entropy splitting for high-order numerical simulation of compressible turbulence, Journal of Computational Physics 178, 307 (2002).

[28] D. J. Lusher and N. Sandham, Assessment of low-dissipative shock-capturing schemes for transitional and turbulent shock interactions, in AIAA Aviation 2019 Forum (2019) p. 3208.

[29] M. H. Carpenter, J. Nordström, and D. Gottlieb, A stable and conservative interface treatment of arbitrary spatial accuracy, Journal of Computational Physics 148, 341 (1999).

[30] J. Williamson, Low-storage Runge-Kutta schemes, Journal of Computational Physics 35, 48 (1980).

[31] C. T. Jacobs, S. P. Jammy, and N. D. Sandham, OpenSBLI: A framework for the automated derivation and parallel execution of finite difference solvers on a range of computer architectures, Journal of Computational Science 18, 12 (2017).

[32] D. J. Lusher, S. P. Jammy, and N. D. Sandham, OpenSBLI: Automated code-generation for heterogeneous computing architectures applied to compressible fluid dynamics on structured grids, Computer Physics Communications , 108063 (2021).

[33] I. Z. Reguly, G. R. Mudalige, M. B. Giles, D. Curran, and S. McIntosh-Smith, The OPS domain specific abstraction for multi-block structured grid computations, in 2014 Fourth International Workshop on Domain-Specific Languages and High-Level Frameworks for High Performance Computing (IEEE, 2014) pp. 58-67.

[34] G. Mudalige, I. Reguly, S. Jammy, C. Jacobs, M. Giles, and N. Sandham, Large-scale performance of a DSL-based multi-block structured-mesh application for direct numerical simulation, Journal of Parallel and Distributed Computing 131, 130 (2019).

[35] S. Laizet, J. Nedić, and J. C. Vassilicos, The spatial origin of $-5 / 3$ spectra in grid-generated turbulence, Physics of Fluids 27, 065115 (2015).

[36] M. Alam and N. D. Sandham, Direct numerical simulation of 'short' laminar separation bubbles with turbulent reattachment, Journal of Fluid Mechanics 410, 1 (2000).

[37] R. Samtaney, D. I. Pullin, and B. Kosović, Direct numerical simulation of decaying compressible turbulence and shocklet statistics, Physics of Fluids 13, 1415 (2001).

[38] J. Wang, T. Gotoh, and T. Watanabe, Shocklet statistics in compressible isotropic turbulence, Physical Review Fluids 2, 023401 (2017). 